\title{
Induction of chronic subclinical systemic inflammation in sprague-dawley rats stimulated by intermittent bolus injection of lipopolysaccharide
}

\begin{abstract}
Chronic subclinical systemic inflammation has a key role in stimulating several chronic conditions associated with cardiovascular diseases, cancer, rheumatoid arthritis, diabetes, and neurodegenerative diseases. Hence, developing in vivo models of chronic subclinical systemic inflammation are essential to the study of the pathophysiology and to measure the immunomodulatory agents involved. Male Sprague-Dawley rats were subjected to intraperitoneal, intermittent injection with saline, or lipopolysaccharide (LPS) $(0.5,1,2 \mathrm{mg} / \mathrm{kg})$ thrice a week for 30 days. Hematological, biochemical, and inflammatory mediators were measured at different timepoints and at the end of the study. The hearts, lungs, kidneys, and livers were harvested for histological evaluation. Significant elevation in peripheral blood leukocyte includes neutrophils, monocytes, and lymphocytes, as well as the neutrophils-tolymphocyte ratio. The pro-inflammatory mediator levels [C-reactive protein, tumor necrosis factor (TNF)- $\alpha$, interleukin (IL)-6, IL-1 $\beta$, and IL-8] along with the biochemical profile (alkaline phosphatase, aspartate aminotransferase, alanine aminotransferase, gamma-glutamyl transferase, creatine kinase, creatinine, and urea) were increased significantly $(\mathrm{P}<0.05)$ and increased the expression of monocyte chemoattractant protein-1 and TNF- $\beta$. The histopathological changes of heart, lung, kidney, and liver tissues revealed degeneration, cellular infiltration of leukocyte in the inflammatory foci and interstitial space, edema, early signs of fibrosis, apoptosis, and necrosis. In conclusion, these results indicate that intermittent exposure to LPS produces chronic subclinical systemic inflammation in multiple organs leading to chronic conditions and supports this model to be a useful preclinical tool for developing immunotherapeutic agents that could prevent, or reduce, chronic inflammatory diseases associated with, or without, bacterial translocation.
\end{abstract}

Keyword: Animal model; Chronic diseases; Cytokines; Lipopolysaccharide; Multiple organs; Systemic low-grade inflammation 